\title{
Phantom study for comparison between computed tomography- and C-Arm computed tomography-guided puncture applied by residents in radiology
}

\section{Phantomstudie zum Vergleich zwischen Computertomografie- und C-Arm-Computertomografie-gesteuertem Punktionsverfahren bei Anwendung durch Weiterbildungsassistenten in der Radiologie}

\section{Authors}

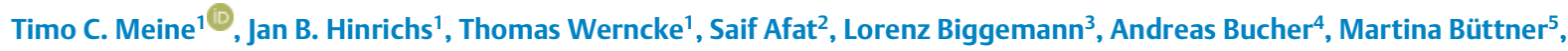
Sara Christner ${ }^{6}$, Ebba Dethlefsen ${ }^{7}$, Hannes Engel ${ }^{8}$, Mirjam Gerwing ${ }^{9}$, Tobias Getzin ${ }^{1}$, Stephanie Gräger ${ }^{10}$, Eva Gresser ${ }^{11}$, Jan-Peter Grunz ${ }^{6}$, Felix Harder ${ }^{12}$, Julius Heidenreich ${ }^{13}$, Lea Hitpaß ${ }^{7}$, Kristina Jakobi ${ }^{14}$, Michael Janisch ${ }^{15}$, Nadja Kocher ${ }^{16}$, Markus Kopp ${ }^{17}$, Simon Lennartz ${ }^{18}$, Ole Martin ${ }^{19}$, Tawfik Moher Alsady ${ }^{10}$, Matthias Pamminger ${ }^{20}$, Frederico Pedersoli ${ }^{7}$, Paula Louise Piechotta21, Natascha Platz Batista da Silva ${ }^{22}$, Marcus Raudner ${ }^{23}$, Sebastian Roehrich ${ }^{23}$, Philipp Schindler , Vincent Schwarze ${ }^{24}$, Danilo Seppelt ${ }^{25}$, Malte M. Sieren ${ }^{26}$, Manuela Spurny ${ }^{27}$, Jitka Starekova ${ }^{28}$, Corinna Storz ${ }^{29}$, Marco Wiesmüller ${ }^{17}$, David Zopfs ${ }^{30}$, Kristina Imeen Ringe ${ }^{1}$, Bernhard C. Meyer ${ }^{1}$, Frank K. Wacker ${ }^{1}$

\section{Affiliations}

1 Institute for Diagnostic and Interventional Radiology, Hannover Medical School, Hannover, Germany

2 Institute for Diagnostic and Interventional Radiology, University Hospital Tübingen, Germany

3 Institute for Diagnostic and Interventional Radiology, University Medical Center Göttingen, Göttingen, Germany

4 Institute of Diagnostic and Interventional Radiology, University Hospital Frankfurt, Germany

5 Clinic for Diagnostic and Interventional Radiology, Ulm University Medical Center, Ulm, Germany

6 Department of Diagnostic and Interventional Radiology, University Hospital Würzburg, Würzburg, Germany

7 Clinic for Diagnostic and Interventional Radiology, University Hospital RWTH Aachen, Germany

8 Department of Radiology, Medical Center - University of Freiburg, Faculty of Medicine, University of Freiburg, Germany

9 Clinic for Radiology, University Hospital Münster, Germany

10 Institute for Diagnostic and Interventional Radiology, Friedrich Schiller University Jena, Germany

11 Department of Radiology, Ludwig Maximilians University Munich, München, Germany

12 Department of Diagnostic and Interventional Radiology, Technical University of Munich, München, Germany

13 Department of Diagnostic and Interventional Radiology, University Hospital Würzburg, Germany

14 Institute for Diagnostic and Interventional Radiology, Rostock University Medical Center, Rostock, Germany

15 Department of Radiology, University Hospital Graz, Austria 16 Department of Diagnostic and Interventional Radiology, Medical Center-University of Freiburg, Germany

17 Institute of Radiology, University Hospitals Erlangen Department of Radiology, Erlangen, Germany
18 Institute for Diagnostic and Interventional Radiology, Faculty of Medicine and University Hospital Cologne, Köln, Germany

19 Department of Diagnostic and Interventional Radiology, University Düsseldorf, Medical Faculty, Düsseldorf, Germany

20 Department of Radiology, Medical University Innsbruck Department of Radiology, Innsbruck, Austria

21 Department of Diagnostic and Interventional Radiology, University Hospital Leipzig, Germany

22 Institute of Diagnostic Radiology, University Hospital Regensburg, Germany

23 University Clinic of Radiology and Nuclear Medicine, General Hospital of the City of Vienna-Hospital of the Medical University of Vienna, Wien, Austria

24 Department of Radiology, Ludwig Maximilians University Munich, München, Germany

25 Department for Diagnostic and Interventional Radiology, University Hospital Carl Gustav Carus Dresden, Germany

26 Clinic for Radiology und Nuclear Medicine, University Medical Center Schleswig-Holstein Lübeck Campus, Lübeck, Germany

27 Department for Diagnostic and Interventional Radiology, University Hospital Heidelberg, Germany

28 Department of Diagnostic and Interventional Radiology, University Hospital Hamburg-Eppendorf Center of Diagnostic, Hamburg, Germany

29 Neuroradiology, University Hospital Freiburg, Germany

30 Institute for Diagnostic and Interventional Radiology, Faculty of Medicine and University Hospital Cologne, Köln, Germany 
Key words

Image Guidance, C-Arm-CT, Phantom Study, Resident in

Training

received 21.02.2021

accepted 27.07.2021

published online 18.11.2021

Bibliography

Fortschr Röntgenstr 2022; 194: 272-280

DOI 10.1055/a-1586-2733

ISSN 1438-9029

(C) 2021. Thieme. All rights reserved.

Georg Thieme Verlag KG, Rüdigerstraße 14,

70469 Stuttgart, Germany

Correspondence

Prof. Frank K Wacker

Institut für Radiologie, Medizinische Hochschule Hannover, Carl-Neuberg-Str. 1, 30625 Hannover, Germany

Tel.: $+49 / 511 / 5323421$

Fax: $+49 / 511 / 5329421$

wacker.frank@mh-hannover.de

\section{ABSTRACT}

Purpose Comparison of puncture deviation and puncture duration between computed tomography (CT)- and C-arm $\mathrm{CT}$ (CACT)-guided puncture performed by residents in training (RiT).

Methods In a cohort of 25 RiTs enrolled in a research training program either CT- or CACT-guided puncture was performed on a phantom. Prior to the experiments, the RiT's level of training, experience playing a musical instrument, video games, and ball sports, and self-assessed manual skills and spatial skills were recorded. Each RiT performed two punctures. The first puncture was performed with a transaxial or single angulated needle path and the second with a single or double angulated needle path. Puncture deviation and puncture duration were compared between the procedures and were correlated with the self-assessments.

Results RiTs in both the CT guidance and CACT guidance groups did not differ with respect to radiologic experience $(p=1)$, angiographic experience $(p=0.415)$, and number of ultrasound-guided puncture procedures $(p=0.483), C T$-guided puncture procedures $(p=0.934)$, and CACT-guided puncture procedures $(p=0.466)$. The puncture duration was significantly longer with CT guidance (without navigation tool) than with CACT guidance with navigation software $(p<0.001)$. There was no significant difference in the puncture duration between the first and second puncture using CT guidance $(p=0.719)$. However, in the case of CACT, the second puncture was significantly faster $(p=0.006)$. Puncture deviations were not different between CT-guided and CACT-guided puncture $(p=0.337)$ and between the first and second puncture of CT-guided and CACT-guided puncture (CT: $p=0.130$; CACT: $p=0.391)$. The self-assessment of manual skills did not correlate with puncture deviation $(p=0.059)$ and puncture duration $(p=0.158)$. The self-assessed spatial skills correlated positively with puncture deviation $(p=0.011)$ but not with puncture duration $(p=0.541)$.

Conclusion The RiTs achieved a puncture deviation that was clinically adequate with respect to their level of training and did not differ between CT-guided and CACT-guided puncture. The puncture duration was shorter when using CACT. CACT guidance with navigation software support has a potentially steeper learning curve. Spatial skills might accelerate the learning of image-guided puncture.

\section{Key Points:}

- The CT-guided and CACT-guided puncture experience of the RiTs selected as part of the program "Researchers for the Future" of the German Roentgen Society was adequate with respect to the level of training.

- Despite the lower collective experience of the RiTs with CACT-guided puncture with navigation software assistance, the learning curve regarding CACT-guided puncture may be faster compared to the CT-guided puncture technique.

- If the needle path is complex, CACT guidance with navigation software assistance might have an advantage over CT guidance.

\section{Citation Format}

- Meine TC, Hinrichs JB, Werncke T et al. Phantom study for comparison between computed tomography- and C-Arm computed tomography-guided puncture applied by residents in radiology. Fortschr Röntgenstr 2022; 194: 272280

\section{ZUSAMMENFASSUNG}

Ziel Vergleich der Punktionsabweichung und -dauer zwischen Computertomografie (CT) - und C-Arm-CT (CACT) -gesteuertem Punktionsverfahren bei Anwendung durch Assistenzärzte in Weiterbildung (AiW).

Material und Methode In einer Kohorte von 25 AiW, die Teil einer wissenschaftlichen Förderung waren, wurden entweder CT- oder CACT-gesteuerte Punktionen an einem Phantom durchgeführt. Vor Beginn wurden der Weiterbildungsstand, die Erfahrung mit Spielen eines Musikinstruments, mit Videospielen und mit Ballsportarten und die Selbsteinschätzung von manueller Geschicklichkeit und räumlichem Denkvermögen abgefragt. Jede/r AiW führte 2 Punktionen durch, wobei die 1. Punktion mit einem transaxialen bzw. einfach angulierten Nadelpfad und die 2. Punktion mit einem einfach bzw. doppelt angulierten Nadelpfad erfolgte. Punktionsabweichung und -dauer wurden zwischen den Verfahren verglichen und mit den Selbsteinschätzungen korreliert.

Ergebnisse Die beiden Gruppen der AiW zeigten keine Unterschiede in der Erfahrung in der Radiologie $(p=1)$, in der Angiografie $(p=0.415)$ und in der Anzahl bereits durchgeführter Punktionen gesteuert durch Ultraschall $(p=0,483)$, CT $(p=0,934)$ und CACT $(p=0,466)$. In der CT (ohne Navigationssoftware) war die Punktionsdauer signifikant länger als mit der CACT-Bildsteuerung mit Navigationssoftware $(p<0,001)$. Bei der Punktionsdauer zeigten sich keine signifi- 
kanten Unterschiede zwischen der 1. und 2. Punktion im CT $(p=0,719)$, während die 2. Punktion mit CACT schneller durchgeführt werden konnte $(p=0,006)$. Die Punktionsabweichung war weder signifikant zwischen CT- und CACT-Bildsteuerung $(p=0,337)$, noch zwischen der 1 . und 2 . Punktion der jeweiligen Verfahren (CT: $p=0,130$; $C A C T: p=0,391)$. Die Selbsteinschätzung der manuellen Geschicklichkeit korrelierte nicht mit der Punktionsabweichung $(p=0,059)$ und -dauer $(p=0,158)$. Das subjektive räumliche Denkvermögen zeigte eine moderate positive Korrelation zur Punktionsabweichung $(p=0,011)$, aber nicht zur -dauer $(p=0,541)$.

Schlussfolgerung Die AiW erreichten eine dem Ausbildungsstand entsprechende, klinisch adäquate Punktionsabweichung unter CT- und CACT-Bildsteuerung. Die CACTgesteuerten Punktionen mit Unterstützung durch Navigationssoftware wurden schneller durchgeführt, und auch die Lernkurve war mit CACT-Bildsteuerung steiler. Räumliches Denkvermögen kann möglicherweise das Erlernen bildgesteuerter Punktionen beschleunigen.

$\begin{array}{ll}\text { ABBREVIATIONS } \\ \text { RiT } & \text { Resident in training } \\ \text { CACT } & \text { C-arm computed tomography } \\ \text { CT } & \text { Computed tomography } \\ \text { DRG } & \text { German Roentgen Society } \\ n & \text { Number } \\ \text { P } & \text { Level of significance } \\ r & \text { Correlation coefficient }\end{array}$

\section{Introduction}

Image-guided diagnostic and therapeutic interventions in radiology have increased in the last 30 years [1]. Image guidance allows exact needle positioning, which is important for ensuring the diagnostic significance of a biopsy and ensuring the effectiveness of local treatment methods [2-4]. Ultrasound-guided and computed tomography-guided puncture are commonly used [5]. The advantage of ultrasound-guided puncture is real-time imaging. The disadvantages include a low penetration depth, particularly in the case of obesity or the superimposition of air, and the dependence on the operator [5]. The CT-guided puncture technique benefits from operator-independent, three-dimensional image information. However, real-time information about the progression of the puncture needle is not available without navigation or is only available on a limited basis in the case of CT fluoroscopy [ 5 , $6]$. There are various options for performing CT-guided interventions. On the one hand, operators can leave the $\mathrm{CT}$ room or can use radiation protective equipment and remain next to the $\mathrm{CT}$ gantry, while computed tomography with minimal slices (typically 3 slices with a slice thickness of $5 \mathrm{~mm}$ ) focused on the puncture tract is performed repeatedly ("quick-and-check"). When the operator leaves the $\mathrm{CT}$ room, there is no radiation exposure. If the examiner remains next to the gantry, the radiation exposure will be negligible. However, the puncture needle must be advanced sequentially and without real-time imaging. On the other hand, real-time imaging is possible with CT fluoroscopy. Using radiation protective equipment, the operator remains in the room. Depending on the technique, the operator's hand even remains on the needle, which is associated with radiation exposure [7].

An alternative to conventional CT is C-arm computed tomography (CACT). The advantage of this puncture method is the com- bination of spatial 3 D CACT information with real-time fluoroscopy information, possibly with the overlay of trajectories [8]. This method reduces the radiation dose [9] and could also make image guidance of complex, double-angulated puncture paths easier compared to CT [10]. The literature specifies a reduction of the effective patient dose for CACT of up to $40 \%$ compared to conventional CT-guided puncture [9]. Depending on the study [10-16], the CACT-guided puncture method even seems to be superior to conventional methods like CT-guided puncture with regard to puncture accuracy.

The puncture deviation and puncture duration of CACT-guided puncture methods performed by experienced interventional radiologists were recently examined by Busser et al. in a phantom study [10]. The training and experience of residents in training (RiT) in radiology with CT-guided and CACT-guided puncture have not yet been studied. However, studies show that the simulation of image-guided methods can improve the learning curve for vascular interventions among RiTs $[17,18]$. The goal of our study was therefore to compare CT image guidance and CACT image guidance among RiTs with limited interventional experience based on puncture deviation and duration in a phantom with different degrees of spatial complexity and to correlate the puncture deviation and duration with the RiTs' manual and spatial skills.

\section{Materials and Methods}

\section{Study participants and covariates}

As part of the structured program "Researchers for the Future" created in 2010 for the targeted promotion of young radiologists by the German Roentgen Society, the Conference of Professors of Radiology, and the Academy for Further and Continuing Education in Radiology, 38 RiTs from university hospitals in Germany and Austria were invited to the Hannover Medical School on March 14 and 15, 2019. 35 RiTs attended. Five RiTs did not actively participate due to organizational reasons and another five due to personal reasons. Thus, a total of 25 RiTs performed punctures in the phantom.

Prior to the event, information regarding the RiTs' level of training was recorded using a questionnaire. The questionnaire included questions regarding professional experience in radiology in years and the number of independently performed puncture 


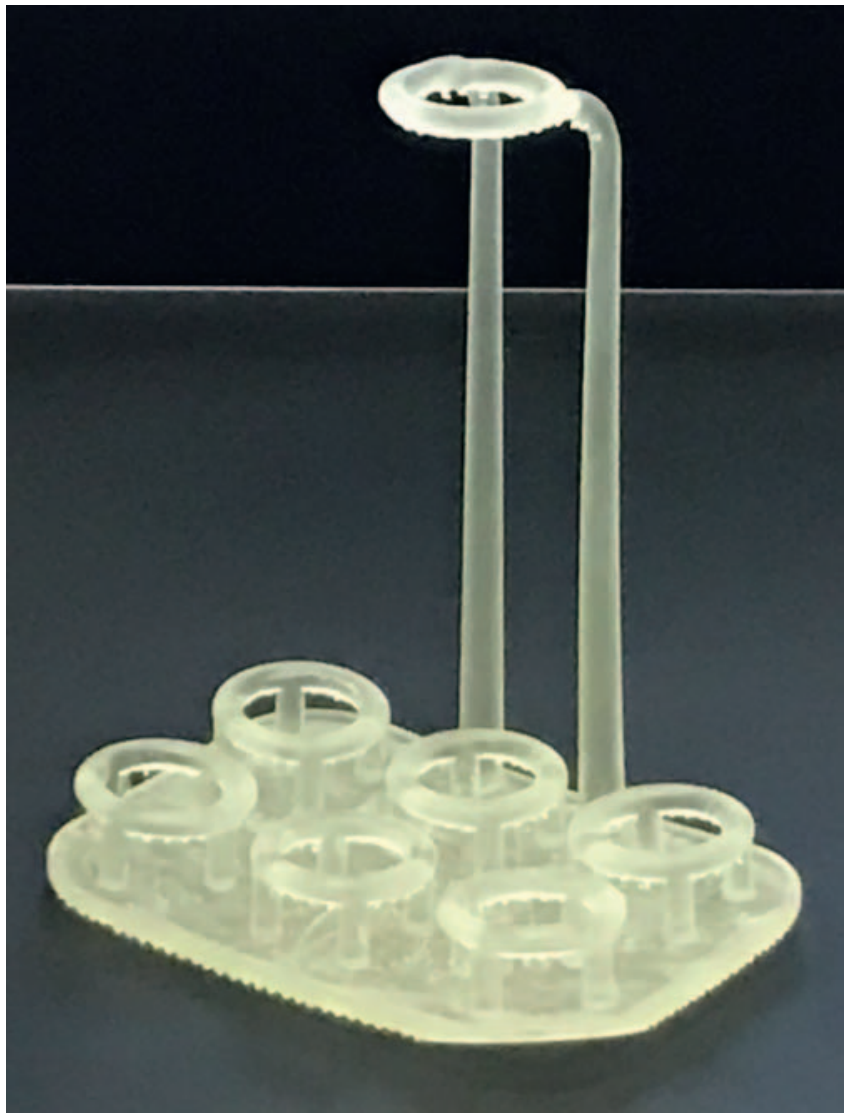

- Fig. 1 Phantom. This figure shows a photograph of the phantom. The phantom has an entry ring and six target rings made of clear resin and was placed in a non-radiopaque gelatin matrix.

procedures (ultrasound-guided, CT-guided), angiography procedures, and CACT-guided puncture procedures. Moreover, the questionnaire included the self-assessment of their manual and spatial skills on a scale of 1-6 (1: very good, 2: good, 3: satisfactory, 4: sufficient, 5: deficient, 6: unsatisfactory) and a qualitative and quantitative assessment of their experience playing a musical instrument, video games, and ball games (type of musical instrument, video game console, and ball sport as well as the number of years of experience).

After a short training session in CT-guided and CACT-guided puncture techniques on the phantom, the RiTs were divided into 6 equal groups with comparable radiology experience to perform the puncture procedures. Two puncture procedures either with CT or CACT image guidance were planned for every RiT a time interval of 30 minutes.

\section{Puncture phantom}

Puncture phantoms were used to analyze the puncture deviation. A three-dimensional printed model with one entry ring and six target rings made of resin (Form 2, clear resin, Formlabs, Somerville, Massachusetts, USA) is embedded in a gelatin matrix (4 liters of distilled water, 350 grams of $7 \%$ gelatin, 35 grams of flour, and 15 milliliters $20 \%$ chlorhexidine) ( $\triangleright$ Fig. 1, 2). After the end of the puncture procedure, the target position was marked with a

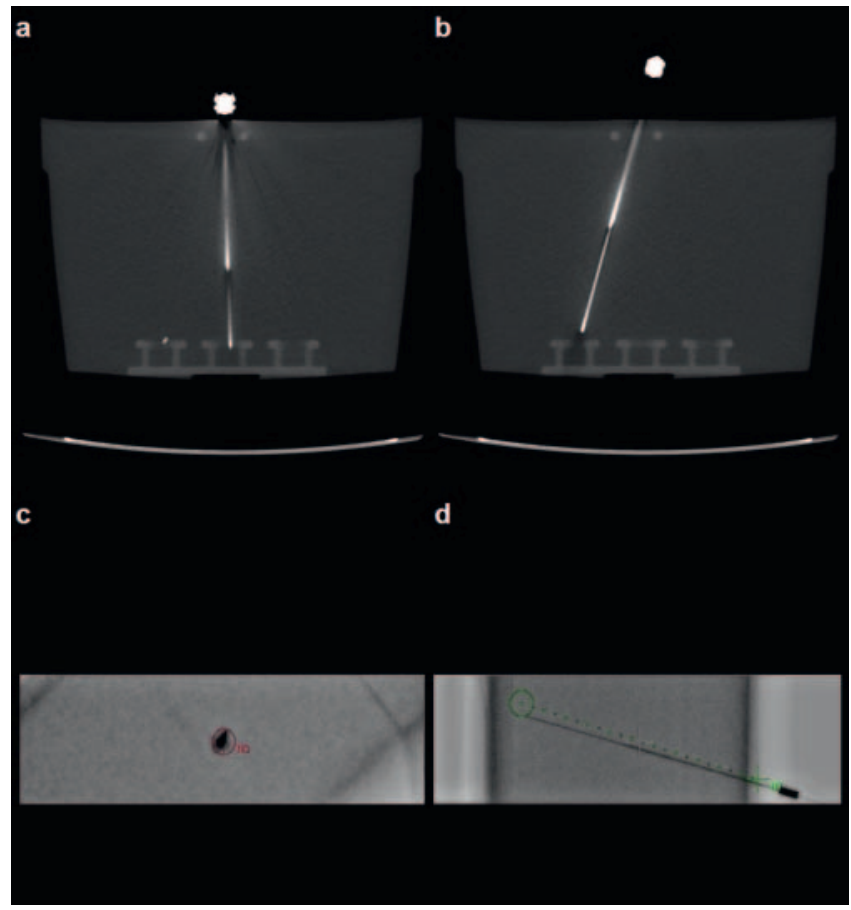

- Fig. 2 Computed tomography-guided and C-arm computed tomography-guided puncture. a The first puncture was planned in a transaxial or single-angulated needle path, shown as an example with CT image control. b The second puncture was carried out in a complex, single-angulated, or double-angulated needle path as shown with CT guidance. c The fluoroscopic image shows the top view of the puncture needle, which is located within the red labeled crosshair of the navigation software ("bulls eye view"). $\mathbf{d}$ In the lateral view, the entire puncture needle is shown in the fluoroscopic image and the needle path of the navigation software is labeled in green (“progression view”).

5-millimeter guidewire fragment (Transend Shapeable Tip, Guidewire with ICE Hydrophilic Coating, $190 \mathrm{~cm}, 0.014$ inch, <0.37 mm; Boston Scientific, Marlborough, Massachusetts, USA) that was advanced through the puncture needle (one-piece angiographic needle with snap-on wing, 18 gauge, $70 \mathrm{~mm}, 0.038$ inch; Cordis, Santa Clara, California, USA/ Chiba Access and Biopsy Needle, 22 gauge, $15 \mathrm{~cm}$; COOK MEDICAL, Bloomington, Indiana, USA). A total of 12 puncture phantoms were available for the 6 groups. After completion of all CT-guided and CACT-guided puncture procedures, the positions of the wire markers in the phantom were detected with a native CT scan (helical, 271 slices, $1.25 \mathrm{~mm}$ slice thickness, 120 kV, 10 mA; GE Lightspeed 16; General Electric, Boston, Massachusetts, USA). The shortest distance from the distal end of the wire marker to the center of the target ring on CT (puncture deviation [mm]) was measured with a ruler function (Visage 7, Visage Imaging $\mathrm{GmbH}$, Berlin, Germany). In addition, the needle placement time (puncture duration [min]) from the start of the first CT or CACT scan to the successful positioning of the wire marker was documented.

\section{Needle placement method}

The puncture was performed either with CT guidance (GE Lightspeed 16; General Electric Healthcare, Chicago, Illinois, USA) or 


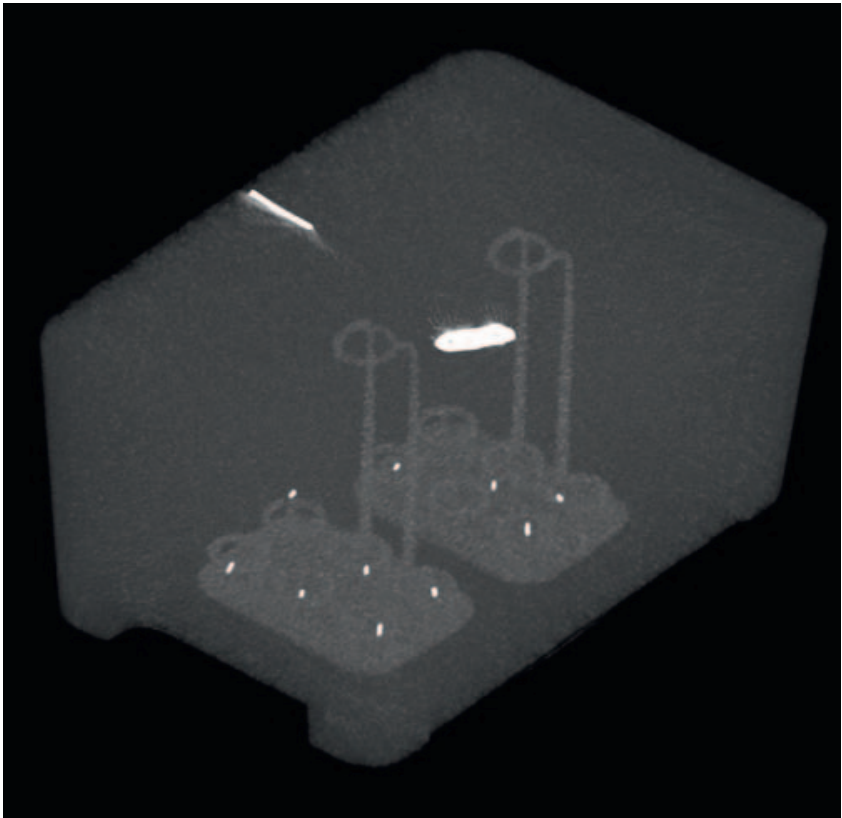

- Fig. 3 Computed tomography of the phantom. This threedimensional reconstruction of the native $\mathrm{CT}$ scan shows the radiopaque markers, which were positioned via CT-guided and C-arm CT-guided puncture. Two paper clips were embedded in the gelatin matrix as additional radiopaque markers for spatial orientation.

CACT guidance (Siemens Pheno; Siemens Healthineers, Erlangen, Germany). The first puncture was performed with a transaxial needle path and the second puncture with a single angulated needle path or the first puncture was performed with single angulated and the second puncture with a double angulated needle path (see $>$ Fig. 2).

\section{CT-guided puncture}

At the start, a native CT scan (helical, 271 slices, $1.25 \mathrm{~mm}$ slice thickness, $120 \mathrm{kV}, 10 \mathrm{~mA}$ ) of the puncture phantom with conventional, radiopaque markers was acquired. The optimal entry point and the needle path to the target were determined. The marker was removed and after placement of the needle at the point of entry, native CT scans in a transaxial direction were repeatedly acquired to check the position of the tip of the needle (transaxial, 5 slices, 2.5-mm slice thickness, $120 \mathrm{kV}, 60 \mathrm{~mA}$ ).

\section{CACT-guided puncture}

For the CACT-guided puncture, the acquisition of a native CACT scan (5 s, 95 projections/s, 397 projections, $90 \mathrm{kV}, 100 \mathrm{~mA}$ ) and reconstruction of a three-dimensional dataset were conducted. The entry point as well as the target point were determined by the person performing the puncture using navigation software. The needle path was calculated automatically. In the first step, the $\mathrm{C}$-arm was automatically positioned in a projection plane perpendicular to the direction of puncture ("bulls eye view") (see $>$ Fig. 2). The intersecting planes of the laser cross hairs integrated in the detector of the angiography system mark the entry point on the phantom and the trajectory. To monitor the progres- sion of the puncture needle in real time via fluoroscopy, the C-arm was automatically moved to a projection plane parallel to the planned needle path ("progression view") (see $>$ Fig. 2). Both view settings could be changed by each resident as needed until the needle or the marker was placed in the target ( Fig. 3).

\section{Evaluation of the phantom study}

In a subsequent questionnaire using SurveyMonkey (www.survey monkey.com, SurveyMonkey Inc., San Mateo, California, USA) the 35 RiTs who were present at the Hannover Medical School were invited to evaluate the phantom study (10 of the RiTs did not actively perform any puncture procedures). The following questions were answered using a Likert scale from 1-5 (1: completely disagree, 2: disagree, 3: neither agree nor disagree, 4: agree, 5: completely agree):

1. Is the phantom generally suitable for CT/CACT-guided puncture training?

2. Can training on a phantom improve patient care?

3. Should CT/CACT-guided puncture training on a phantom be part of the RiT program?

4. Is the currently offered training (e. g., at conventions, in workshops, or in your own department) regarding CT/CACT-guided interventions in Germany and Austria sufficient (prior to the pandemic)?

\section{Statistical analysis}

The information provided by the RiTs in the questionnaire was recorded in the categories described above with the mean value and standard deviation. The level of training of the RiTs who performed puncture with CT image guidance was compared with that of the RiTs who performed puncture with CACT image guidance. The puncture deviation and puncture duration were compared between the methods and between the first and second puncture. The puncture deviation and puncture duration were then correlated with the self-assessment regarding manual and spatial skills to detect a potential difference and any advantage for learning image-guided methods. The evaluation results were documented with the number of responses on the Likert scale.

The statistical evaluation was performed with the R 3.6.2 statistical computation system (https://www.r-project.org). In the case of non-parametric distribution analyzed with the ShapiroWilk test, the Mann-Whitney $U$ test for independent samples was used for the comparison between CT and CACT image guidance. The Wilcoxon test for independent samples was performed for the comparison between the first and second puncture within a group. One participant, who only performed the first puncture in the available time, was excluded from the independent comparison between the first and second puncture within the group with $\mathrm{CT}$ image guidance. The correlation was analyzed with the Spearman rank correlation coefficient $(r)$. Two-sided testing was performed with a significance level of $p<0.05$. 
- Table 1 Residents in training in radiology.

\begin{tabular}{|c|c|c|c|}
\hline & $\begin{array}{l}\text { CT } \\
(n=11)\end{array}$ & $\begin{array}{l}\text { CACT } \\
(n=14)\end{array}$ & p-value \\
\hline professional experience in radiology (years) & $3 \pm 1$ & $3 \pm 1$ & 1 \\
\hline number of conventional CT-guided puncture procedures & $36 \pm 47$ & $35 \pm 42$ & 0.934 \\
\hline number of ultrasound-guided puncture procedures & $14 \pm 25$ & $14 \pm 40$ & 0.483 \\
\hline number of angiography procedures & $11 \pm 19$ & $45 \pm 89$ & 0.415 \\
\hline number of CACT-guided puncture procedures & $5 \pm 15$ & $11 \pm 39$ & 0.466 \\
\hline
\end{tabular}

This table shows the professional experience of residents in training in radiology who performed either computed tomography (CT)-guided or C-arm computed tomography (CACT)-guided puncture. The mean values and standard deviation as well as the $\mathrm{p}$-value of the Mann-Whitney-U test are given. Abbreviations: $\mathrm{n}=$ number of residents in training.

- Table 2 Target deviation and puncture duration between computed tomography-guided and C-arm computed tomography-guided puncture.

\begin{tabular}{|c|c|c|c|}
\hline & $\begin{array}{l}\text { CT } \\
(n=21)\end{array}$ & $\begin{array}{l}\text { CACT } \\
(n=28)\end{array}$ & p-value \\
\hline puncture deviation [mm] & $7.2 \pm 3.3$ & $7.9 \pm 3.3$ & 0.337 \\
\hline puncture duration [min] & $11 \pm 11$ & $6 \pm 2$ & $<0.001$ \\
\hline
\end{tabular}

This table lists the target deviation and puncture duration by the residents who performed either computed tomography (CT)-guided or $\mathrm{C}$-arm computed tomography (CACT)-guided puncture. Mean values and standard deviation as well as the $\mathrm{p}$-value of the Mann-Whitney-U test are given. Abbreviations: $\min =$ minute(s), $\mathrm{mm}=$ millimeter(s) and $\mathrm{n}=$ number of values.

\section{Results}

\section{Study participants and covariates}

The average professional experience in radiology per RiT was $3 \pm 1$ year. The number of already performed puncture procedures per RiT was $14 \pm 34$ for ultrasound-guided puncture, $36 \pm 44$ for CT-guided puncture, $30 \pm 70$ for angiography, and $8 \pm 31$ for CACT-guided puncture. In the self-assessment, both manual and spatial skills were assigned a value of $2 \pm 1$. 18 RiTs had experience playing a musical instrument, 20 playing video games, and 17 playing ball sports. The accordion, cello, electric bass, guitar, clarinet, piano, organ, German flute, and violin were listed as the musical instruments. Basketball, soccer, handball, squash, tennis, table tennis, and volleyball were specified as the types of ball sport. Neither the level of training nor the experience with punctures was statistically different between the RiTs that performed CT-guided puncture and those that performed CACT-guided puncture ( $\triangleright$ Table 1$)$.

\section{Puncture deviation in the phantom}

The difference in the puncture deviation between CT and CACT was not significant $(7.2 \pm 3.3 \mathrm{~mm}$ and $7.9 \pm 3.3 \mathrm{~mm})(p=0.337)$. There was also no statistical difference between the first and second puncture in the CT group $(6.4 \pm 2.7 \mathrm{~mm}$ and $8.5 \pm 3.5 \mathrm{~mm}$; $\mathrm{p}=0.130)$ and in the CACT group $(8.3 \pm 4.2 \mathrm{~mm}$ and $7.6 \pm 2.2 \mathrm{~mm}$; $\mathrm{p}=0.391)$. The results are provided in detail in $>$ Table 2, 3 .

\section{Puncture duration on the phantom}

The puncture duration of CACT-guided puncture ( $6 \pm 2 \mathrm{~min})$ was significantly shorter than that of CT-guided puncture $(11 \pm 11 \mathrm{~min})(p<0.001)$. In the case of CACT, the second, more difficult puncture was performed more quickly $(5 \pm 2 \mathrm{~min})$ than the first puncture $(7 \pm 2 \mathrm{~min})(p=0.006)$. In the case of CT-guided puncture, there was no statistical difference between the first and second puncture ( $13 \pm 17 \mathrm{~min}$ compared to $9 \pm 3 \mathrm{~min})(p=0.719)$. The results are shown in $>$ Table 2, 3.

\section{Influence of the self-assessment}

The self-assessment of manual skills did not correlate with the puncture deviation $(r:+0.271 ; p=0.059)$ and the puncture duration ( $r$ : $-0.204 ; p=0.158)$. There was a significant correlation between the self-assessment of spatial skills and puncture deviation ( $r$ : $-0.089 ; p=0.541)$ but not between spatial skills and puncture duration $(r:-0.089 ; p=0.541)$. The results are shown in Table 4.

\section{Evaluation of the phantom study}

Willingness to participate in the subsequent questionnaire regarding the phantom study was high (33 of 35 RiTs (94\%)). 
- Table 3 Target deviation and puncture duration between the first and second puncture of the computed tomography-guided or C-arm computed tomography-guided puncture.

\begin{tabular}{|c|c|c|c|}
\hline & first puncture & second puncture & p-value \\
\hline CT & $n=10$ & $n=10$ & \\
\hline puncture deviation [mm] & $6.4 \pm 2.7$ & $8.5 \pm 3.5$ & 0.130 \\
\hline puncture duration [min] & $13 \pm 17$ & $9 \pm 3$ & 0.719 \\
\hline САCT & $n=14$ & $n=14$ & \\
\hline puncture deviation [mm] & $8.3 \pm 4.2$ & $7.6 \pm 2.2$ & 0.391 \\
\hline puncture duration [min] & $7 \pm 2$ & $5 \pm 2$ & 0.006 \\
\hline
\end{tabular}

- Table 4 Impact of self-assessment on target deviation and puncture duration in the phantom.

\begin{tabular}{l|l|l|}
\hline \begin{tabular}{l|l} 
correlation & r-value \\
(n=49)
\end{tabular} & p-value \\
\hline \begin{tabular}{l|l} 
puncture deviation/spatial skills \\
puncture deviation/manual skills
\end{tabular} & +0.356 & 0.011 \\
\hline puncture duration/spatial skills & +0.271 & 0.059 \\
\hline puncture duration/manual skills & -0.089 & 0.541 \\
\hline
\end{tabular}

In total, $97 \%$ of the RiTs agreed that the phantom is generally suitable for CT/CACT-guided puncture training (number of responses on the Likert scale: $1=0,2=0,3=1,4=14,5=18$ ) and that patient care can be improved by training on a phantom (Likert scale response distribution: $1=0,2=0,3=1,4=10,5=22$ ). $91 \%$ of the RiTs found that CT/CACT-guided puncture training on a phantom should be part the RiT program (Likert scale response distribution: $1=0,2=2,3=1,4=5,5=25$ ). In contrast, $27 \%$ of the RiTs neither agreed nor disagreed and $61 \%$ disagreed that the currently offered CT/CACT-guided intervention training in Germany and Austria is sufficient (Likert scale response distribution: $1=5,2=15,3=9,4=2,5=2$ ).

\section{Discussion}

In our study, RiTs from university radiology departments from all over Germany participating in the "Researchers for the Future" program of the German Roentgen Society performed puncture procedures. On average, the RiTs selected by the individual university hospitals were in the third year of their RiT program and had already performed the number of non-vascular interventions required by the Specialty Training Regulations [19]. Based on clinical practice, experience with CT-guided puncture is greater than with CACT-guided puncture as expected. Overall, a slightly greater puncture deviation from the target of approx. $7 \mathrm{~mm}$ was seen in our study in the CT group and the CACT group compared to the literature, e. $\mathrm{g}$. a deviation of $3 \mathrm{~mm}$ among experienced interventional radiologists was reported in the phantom study by Busser et al. and between $3 \mathrm{~mm}$ and $12 \mathrm{~mm}$ in clinical practice $[10,20,21]$. However, since the RiTs performed puncture procedures after a brief introduction to an unfamiliar environment in our phantom study, the puncture deviation is not unexpected and is clinically acceptable in many cases.

The puncture time for CACT-guided puncture ( $6 \pm 2 \mathrm{~min}$ ) was significantly shorter than for CT-guided puncture (11 $\pm 11 \mathrm{~min}$.) This could be due to the workflow since the RiTs wore radiation protective clothing and remained in the angiography room during CACT-guided puncture while the RiT of the CT group left the examination room during CT-guided puncture. Moreover, CACT puncture guidance is supported by a navigation tool while no software support was available for $\mathrm{CT}$. This navigation software seems to be intuitive even for people with minimal experience performing puncture procedures since the second and significantly more difficult CACT-guided puncture was faster than the first puncture. This learning effect was lower and not statistically significant in the CT group without a navigation tool. The learning effect regarding puncture deviation was also seen in the phantom study 
by Busser et al. even among experienced interventional radiologists [10]. Therefore, our study shows that navigated CACTguided puncture allows a steep learning curve even among inexperienced RiTs in radiology. CT-guided puncture would presumably also benefit from software support provided that its use is similarly intuitive. Hence, CT-guided and CACT-guided interventions using modern navigation tools should be simulated and tested on phantoms and be offered on a more intensive basis than a training unit during the RiT program to prepare RiTs with minimal experience for clinical application.

There was no significant correlation between the self-assessment of manual skills and the puncture deviation or puncture duration. There was also no correlation between the selfassessment of spatial skills and the puncture duration. However, there was a moderate positive correlation between spatial skills and puncture deviation. The last result highlights the relevance of spatial skills for learning image-guided interventions. Although this skill can be different in people, it can be improved by training [22]. Therefore, spatial skills training, for example, using a phantom or simulator could contribute to a steeper learning curve for image-guided interventions. This has already been shown by other studies for endovascular interventions. For example, the fluoroscopy time and the intervention duration of subsequent interventions in the clinical routine could be significantly reduced by simulator training, e. g. stent implantation in the internal carotid artery or diagnostic coronary angiography $[17,18]$. Since most RiTs had two or more hobbies requiring manual skills, a further statistical evaluation was not possible due to the lack of a group of RiTs without hobbies.

The questionnaire regarding the phantom study had a high response rate (94\%) and a uniform response pattern resulting in a clear result. The phantom used in the study was rated as suitable for CT/CACT-guided puncture training and the RiTs felt that the training on a phantom could also help to improve patient care. Even though training on a phantom is not currently part of the RiT program, the RiTs felt that it should be as in other professional groups like pilot training. The RiTs consider the training options for non-vascular interventions on a phantom currently available in Germany and Austria as insufficient.

Our phantom study has some limitations. The assessment of manual and spatial skills was subjective, but could be provided objectively by tests. The use of the "quick-and-check" technique for CT and the navigation software for CACT guidance limits the ability to compare the methods even though this corresponds to the clinical routine since tools are not used at many institutions for CT-guided biopsies and drainage procedures. In contrast, the CACT-guided technique is hardly feasible without navigation. Moreover, the radiation exposure in CACT-guided puncture techniques with overlay is $40 \%$ lower compared to conventional CT-guided puncture [9]. The last and most important limitation is the low number of experiments and participants. Although the average puncture experience of the RiTs corresponded to their level of training, the group was very heterogenous and that explains the high deviation of values and corresponding limitations of the statistical analysis. Unfortunately, the time in the "Researchers for the Future" program was limited so that the number of puncture procedures could not be increased and the
RiTs did not have time to perform the two puncture techniques. Our results and the questionnaire can be used as the basis for further studies with a corresponding number of cases and study design in order to improve simulators and at the same time to further evaluate the advantages and disadvantages of puncture methods, particularly for those with limited experience performing puncture procedures.

\section{Funding}

Deutsche Röntgengesellschaft e. V. (Forscher-für-die-Zukunft)

\section{CLINICAL RELEVANCE OF THE STUDY}

- In the group of RiTs selected from university radiology departments as part of the "Researchers for the Future" program, the experience with CT-guided puncture corresponds to the standard defined by the Specialty Training Regulations.

- Although experience with CACT image guidance is significantly lower, CACT with software support seems to have a steeper learning curve than conventional CT.

- The RiTs rated their skills high and achieved accuracy in the study corresponding to their level of training.

\section{Conflict of Interest}

Lorenz Biggemann: L. B. declares travel grant from Siemens Healthineers and speakers honorarium from Bristol Myer-Squibb unrelated to this project.

Jan -Peter Grunz: J.-P. G. declares employment as "Research Consultant" at Siemens Healthineers unrelated to this project.

Markus Kopp: M. B. declares participation at the Siemens Healthineers speakers' bureau unrelated to this project.

Simon Lennartz: S. L. declares institutional research support from Philips unrelated to this project.

Timo C. Meine: T.C.M. declares passive participation at the BTG TheraSphere ${ }^{T M}$ DACH Summit 2018 unrelated to this to this project. Bernhard C. Meyer: B.C.M. declares relationships with Siemens Healthcare and ProMedicus (outside the submitted work).

Frank K. Wacker: F. K. W. declares relationships with Siemens Healthcare and ProMedicus (outside the submitted work).

David Zopfs: D. Z. declares institutional research support from Philips Healthcare unrelated to this project.

\section{References}

[1] Helmberger T, Martí-Bonmatí L, Pereira P et al. Radiologists' leading position in image-guided therapy. Insights Imaging 2013; 4: 1-7. doi:10.1007/s13244-012-0213-9

[2] Ahmed M, Brace CL, Lee FT et al. Principles of and Advances in Percutaneous Ablation. Radiology 2011; 258: 351-369. doi:10.1148/ radiol. 10081634

[3] Goldberg SN, Gazelle GS, Mueller PR. Thermal Ablation Therapy for Focal Malignancy: A Unified Approach to Underlying Principles, Techniques, and Diagnostic Imaging Guidance. American Journal of Roentgenology 2000; 174: 323-331. doi:10.2214/ajr.174.2.1740323

[4] Zhao G, Shi X, Sun W et al. Factors affecting the accuracy and safety of computed tomography-guided biopsy of intrapulmonary solitary nodules 
$\leq 30 \mathrm{~mm}$ in a retrospective study of 155 patients. Experimental and Therapeutic Medicine 2017; 13: 1986-1992. doi:10.3892/etm.2017.4179

[5] Charboneau JW, Reading CC, Welch TJ. CT and sonographically guided needle biopsy: current techniques and new innovations. American Journal of Roentgenology 1990; 154: 1-10. doi:10.2214/ajr.154.1.2104689

[6] Silverman SG, Tuncali K, Adams DF et al. CT Fluoroscopy-guided Abdominal Interventions: Techniques, Results, and Radiation Exposure. Radiology 1999; 212: 673-681. doi:10.1148/radiology.212.3. r99se36673

[7] Sarti M, Brehmer WP, Gay SB. Low-Dose Techniques in CT-guided Interventions. RadioGraphics 2012; 32: 1109-1119. doi:10.1148/ rg.324115072

[8] Racadio JM, Babic D, Homan R et al. Live 3D Guidance in the Interventional Radiology Suite. American Journal of Roentgenology 2007; 189 : W357-W364. doi:10.2214/Am J Roentgenol.07.2469

[9] Braak SJ, van Strijen MJL, van Es HW et al. Effective Dose during Needle Interventions: Cone-beam CT Guidance Compared with Conventional CT Guidance. Journal of Vascular and Interventional Radiology 2011; 22: 455-461. doi:10.1016/j.jvir.2011.02.011

[10] Busser WMH, Braak S], Fütterer J] et al. Cone beam CT guidance provides superior accuracy for complex needle paths compared with CT guidance. BJR 2013; 86: 20130310 doi:10.1259/bjr.20130310

[11] Jin KN, Park CM, Goo JM et al. Initial experience of percutaneous transthoracic needle biopsy of lung nodules using $\mathrm{C}$-arm cone-beam CT systems. Eur Radiol 2010; 20: 2108-2115. doi:10.1007/s00330-010$1783-x$

[12] Choo JY, Park CM, Lee NK et al. Percutaneous transthoracic needle biopsy of small $(\leq 1 \mathrm{~cm})$ lung nodules under $\mathrm{C}$-arm cone-beam CT virtual navigation guidance. Eur Radiol 2013; 23: 712-719. doi:10.1007| s00330-012-2644-6

[13] Higashihara H, Osuga K, Onishi H et al. Diagnostic accuracy of C-arm CT during selective transcatheter angiography for hepatocellular carcinoma: comparison with intravenous contrast-enhanced, biphasic, dynamic MDCT. Eur Radiol 2012; 22: 872-879. doi:10.1007/s00330011-2324-y
[14] Braak S], van Melick HHE, Onaca MG et al. 3D cone-beam CT guidance, a novel technique in renal biopsy-results in 41 patients with suspected renal masses. Eur Radiol 2012; 22: 2547-2552. doi:10.1007/s00330012-2498-y

[15] Lee WJ, Chong S, Seo JS et al. Transthoracic fine-needle aspiration biopsy of the lungs using a C-arm cone-beam CT system: diagnostic accuracy and post-procedural complications. BJR 2012; 85: e217-e222. doi:10.1259/bjr/64727750

[16] Hwang HS, Chung MJ, Lee JW et al. C-Arm Cone-Beam CT-Guided Percutaneous Transthoracic Lung Biopsy: Usefulness in Evaluation of Small Pulmonary Nodules. American Journal of Roentgenology 2010; 195: W400-W407. doi:10.2214/Am J Roentgenol.09.3963

[17] Gosling AF, Kendrick DE, Kim AH et al. Simulation of carotid artery stenting reduces training procedure and fluoroscopy times. Journal of Vascular Surgery 2017; 66: 298-306. doi:10.1016/j.jvs.2016.11.066

[18] Prenner SB, Wayne DB, Sweis RN et al. Simulation-based education leads to decreased use of fluoroscopy in diagnostic coronary angiography. Catheter Cardiovasc Interv 2018; 91: 1054-1059. doi:10.1002/ ccd. 27203

[19] Bundesärztekammer A der deutschen Ä. (Muster-)Weiterbildungsordnung 2018. Im Internet (Stand: 01.08.2020): 2020 https://www.bunde saerztekammer.de/fileadmin/user_upload/downloads/pdf-Ordner/Wei terbildung/20200428_MWBO_2018.pdf

[20] Abi-Jaoudeh N, Kruecker J, Kadoury S et al. Multimodality image fusionguided procedures: technique, accuracy, and applications. Cardiovasc Intervent Radiol 2012; 35: 986-998. doi:10.1007/s00270-012-0446-5

[21] Abi-Jaoudeh N, Fisher T, Jacobus J et al. Prospective Randomized Trial for Image-Guided Biopsy Using Cone-Beam CT Navigation Compared with Conventional CT. J Vasc Interv Radiol 2016; 27: 1342-1349. doi:10.1016/j.jvir.2016.05.034

[22] Wiedenbauer G, Jansen-Osmann P. Manual training of mental rotation in children. Learning and Instruction 2008; 18: 30-41. doi:10.1016/ j.learninstruc.2006.09.009 